\title{
Gender Differences in Korean Patients with Obstructive Sleep Apnea
}

\author{
So Young Pyun, MD, $\mathrm{PhD}^{1 *}$, Su Jung Choi, RN, APN, PhD ${ }^{2 *}$, Hyunjin Jo, $\mathrm{MD}^{3}$, \\ Yoonha Hwang, $\mathrm{MD}^{3}$, Jae Wook Cho, MD, $\mathrm{PhD}^{4}$, Eun Yeon Joo, MD, $\mathrm{PhD}^{3}$ \\ 'Department of Neurology, LTG Neuro Medical Center, Seoul, Korea \\ ²Department of Nursing, Samsung Medical Center, Graduate School of Clinical Nursing Science, Sunkyunkwan University, Seoul, Korea \\ ${ }^{3}$ Department of Neurology, Neuroscience Center, Samsung Biomedical Research Institute, Samsung Medical Center, \\ Sungkyunkwan University School of Medicine, Seoul, Korea \\ ${ }^{4}$ Department of Neurology, Research Institute for Convergence of Biomedical Science and Technology, Pusan National University Yangsan Hospital, \\ Yangsan, Korea
}

\author{
Received: April 10, 2020 \\ Revised: June 9, 2020 \\ Accepted: July 20, 2020 \\ Correspondence \\ Eun Yeon Joo, MD, PhD \\ Department of Neurology, \\ Samsung Medical Center, \\ Sungkyunkwan University School of Medicine, \\ 81 Irwon-ro, Gangnam-gu, Seoul 06351, Korea \\ Tel +82-2-3410-3599 \\ Fax +82-2-3410-0052 \\ E-mail ejoo@skku.edu \\ *These authors contributed equally to this work. \\ ORCID \\ So Young Pyun \\ https://orcid.org/0000-0002-6306-164X \\ Su Jung Choi \\ https://orcid.org/0000-0003-2171-7441 \\ Hyunjin Jo \\ https://orcid.org/0000-0001-9563-1849 \\ Yoonha Hwang \\ https://orcid.org/0000-0002-2624-9336 \\ Jae Wook Cho \\ https://orcid.org/0000-0002-2742-9136 \\ Eun Yeon Joo \\ https://orcid.org/0000-0003-1233-959X
}

(C) This is an Open Access article distributed under the terms of the Creative Commons Attribution Non-Commercial License (https://creativecommons.org/licenses/by-nc/4.0) which permits unrestricted non-commercial use, distribution, and reproduction in any medium, provided the original work is properly cited.
Background and Objective Our objective in this study was to investigate gender-specific differences in demographic factors, clinical presenting symptoms, medical comorbidities, and sleeprelated parameters in patients newly diagnosed with obstructive sleep apnea (OSA).

Methods We enrolled patients who were more than 30 years old, had undergone overnight polysomnography, and had filled out the questionnaires, including demographics, medical comorbidities, and sleep-related symptoms.

Results From December 2014 to August 2017, 1224 patients (female, $n=277,22.6 \%$ ) were newly diagnosed with OSA. Female patients were significantly older than males (59.62 years vs. 52.75 years, $\mathrm{p}<0.001)$. Alcohol consumption and current smoking were more common in males $(\mathrm{p}<$ 0.001). Non-specific symptoms of OSA, such as insomnia-related symptoms, loss of energy, and subjective poor sleep, were observed more frequently in females. Females reported more depressive moods (Beck Depression Inventory-II 15.58) than males did (12.17). Males complained about OSA-specific symptoms, such as snoring, witnessed apnea, and daytime sleepiness, more frequently than females did. The mean apnea-hypopnea index (AHI) was much lower in females $(26.25 / \mathrm{h}$ vs. $33.36 / \mathrm{h})$, but AHI during rapid eye movement sleep was similar for the two groups $(33.55 / \mathrm{h}$ vs. $32.76 / \mathrm{h})$. Sleep latency was longer $(15.45 \mathrm{~min}$ vs. $10.11 \mathrm{~min})$ and spontaneous arousal index $(4.48 / \mathrm{h}$ vs. $3.48 / \mathrm{h}$ ) was more frequent in females.

Conclusions Females have a different OSA phenotype from males in terms of demographics, clinical manifestations, and sleep parameters of polysomnography. Thus, clinicians should comprehend the different clinical phenotype of female OSA and detect earlier unrecognized OSA in females.

Sleep Med Res 2020;11(2):121-128

Key Words Obstructive sleep apnea, Sex, Sleep initiation and maintenance disorders, Polysomnography.

\section{INTRODUCTION}

Obstructive sleep apnea (OSA) is considered to be a disorder that occurs predominantly in males, possibly because of differences in clinical symptoms and accordingly different rates of using sleep clinics. The incidence of OSA established in the apnea-hypopnea index (AHI) $\geq 5 / \mathrm{h}$ was $9-37 \%$ in males and $4-16 \%$ in females in eleven epidemiological studies about various age groups, using different methods [1-6]. However, it is now widely recognized that OSA in females is not as rare as was originally believed [1]. The Wisconsin Sleep cohort study revealed that OSA occurs in females as much as in males after menopause, and its peak was in the fifth 
and sixth decades [7]. The male-to-female prevalence ratio is 2-3:1 in the general population, but an 8:1 or greater ratio is observed in clinical settings [8]. This disparity indicates that OSA is clinically under-recognized in females by primary physicians. Explanations for male predominance include gender differences in upper airway anatomy and collapsibility [9], bodyfat distribution [10], and hormonal effects [11]. Compared to the distinctions in pathophysiology, gender differences in clinical characteristics and polysomnography findings, which delay OSA diagnosis and treatment, have not been discussed in Korean patients as much as in many western countries. The association between OSA and vascular disease has been shown to be detrimental, supporting the development of cardiovascular disorders, stroke, metabolic syndrome, cognitive impairment, and car accidents caused by daytime somnolence [12]. Therefore, early diagnosis and appropriate intervention for OSA is recommended to improve quality of life and to reduce the risk of associated morbidity and mortality. Accordingly, we evaluated the difference in demographics, clinical presentation, and polysomnographic findings between Korean female and male patients referred to the single tertiary hospital.

\section{METHODS}

\section{Subjects}

We used a sleep laboratory database to identify all subjects who were newly diagnosed with OSA by full-night polysomnography (PSG) from December 2014 to August 2017 at the sleep clinic of Samsung Medical Center. Inclusion criteria were ages more than 30 years and AHI of $\geq 5 / \mathrm{h}$. All subjects were Korean-speaking adults and had completed self-report questionnaires before the sleep study. Exclusion criteria were as follows:

- patients who had been previously diagnosed with OSA or had been receiving treatment for OSA such as continuous positive airway pressure or tonsillectomy or had accompanied central sleep apnea; other sleep disorders, such as narcolepsy, rapid eye movement (REM) sleep behavior disorder (RBD), shift-work sleep disorder (SWSD);

- other neurological disease (neurodegenerative disease, such as Parkinson's disease and dementia);

- incomplete sleep questionnaires.

Of the 1440 subjects, those with previously treated as OSA, $\mathrm{n}=108$; with combined sleep disorders, $\mathrm{n}=52$ (RBD 31, Narcolepsy 20, SWSD 1); or with incomplete questionnaires, $\mathrm{n}=$ 56. Finally, 1224 subjects (male $=947$, female $=277$ ) were included in the study for the final analyses. The study protocol was approved by the Ethics Committee of Samsung Medical Center (IRB No. 2020-03-193).

PSG studies were recorded during one-night observation with standard electrodes and sensors using Remlogic (Embla Systems, Denver, CO, USA). Electroencephalography electrodes were ap- plied at C3-A2, C4-A1, F3-A2, F4-A1, O3-A2, and O2-A1, and four electrooculography electrodes were applied at both lateral sides of the eyes, superior to the eyes, and inferior to the eyes to record horizontal and vertical eye movements. Chin and both anterior tibialis electromyogram and electrocardiography sensors were applied. Two plethysmography belts were used to monitor thoracic and abdominal movements. Nasal and oral airflow was measured with a nasal pressure transducer and a thermistor. Oxygen saturation was measured by pulse oximetry via the index finger. Synchronized video monitoring was used to monitor abnormal breathing or movements during sleep. We collected data from PSG as sleep parameters [total sleep time (TST), sleep latency, wakefulness after sleep onset (WASO), sleep efficiency (SE), sleep stage, and AHI]. Apnea was defined as a complete cessation of airflow for at least $10 \mathrm{~s}$, whereas hypopnea was defined as a moderate reduction in airflow $(>30 \%)$ for at least $10 \mathrm{~s}$ with oxygen desaturation $(\geq 4 \%)$ or arousal. AHI was the average number of apneas and hypopneas in an hour of sleep [13]. We categorized OSA as mild $(5 \leq \mathrm{AHI}<15 / \mathrm{h})$, moderate ( $15 \leq$ AHI $<30 / h$ ), or severe (AHI $\geq 30 / h$ ).

\section{Questionnaires}

Data was collected with surveys about basic demographic factors [age, sex, education level, body mass index (BMI), alcohol, smoking, and sedative drug-use history], and medical comorbidities (hypertension, diabetes mellitus, hyperlipidemia, cardiovascular, cerebrovascular, respiratory disease, and otolaryngologic disease). Self-reported sleep-related symptoms (snoring, witnessed apnea, difficulty falling asleep, difficulty maintaining sleep, early morning awakening, loss of energy, and irritability) consisted of each item per symptom, which was rated on a Likert Scale of 0 (absence of symptom) to 4 (very severe). If more than two points (moderate or more) were checked in each item, it was considered to be a symptom. We also collected the score of the Epworth Sleepiness Scale (ESS) [14], insomnia severity index [15], Pittsburgh sleep quality index (PSQI) [16], and Beck Depression Inventory-II (BDI-II) [17]. Subjects with an ESS score $>10$ were considered as having clinically significant daytime sleepiness [14].

\section{Statistical Analysis}

All statistical analyses were conducted using the Statistical Package for Social Science (SPSS) for Windows, version 18.0 (SPSS, Chicago, IL, USA). Statistical significance level was set at $\mathrm{p}<0.05$. We analyzed all continuous variables using the independent t-test or Mann-Whitney test, and analyzed categorical variables using Pearson's chi-square tests or Fisher's exact test. 


\section{RESULTS}

\section{Demographics of Female and Male OSA Patients}

The overall male: female ratio for OSA was 3.4:1 (947 vs. 277). Female patients were significantly older than male patients (59.62 \pm 11.16 years vs. $52.75 \pm 11.46$ years, $\mathrm{p}<0.001)$ and slightly lighter (BMI $25.01 \pm 3.96 \mathrm{~kg} / \mathrm{m}^{2}$ vs. $25.77 \pm 3.27 \mathrm{~kg} / \mathrm{m}^{2}$, respectively, $\mathrm{p}=0.004$ ). Females and males differed in age distribution. In females, the proportion of patients over 50 years in age was $82.3 \%$. In contrast, males in their 50's were the largest group, followed by those in their 40's, which also demonstrated relatively even distribution in all age groups. Interestingly, the percentage of OSA prevalence in those over 70 years was higher in females than in males (18.8\% vs. $7.0 \%$ ) (Table 1 ). When compared with age distribution, the BMI was not significantly different between the two groups (Fig. 1A, Table 2). The rates of alcohol use (71.8\% vs. $23.2 \%, \mathrm{p}<0.001)$ and current smoking $(20.9 \%$ vs. $2.6 \%, \mathrm{p}<0.001)$ were higher in males with OSA, whereas females took more hypnotics and sedative drugs (22.3\% vs. $9.3 \%$, $\mathrm{p}<0.001)$. The presence of comorbid diseases was similar between males and females (Table 1).

\section{Clinical Symptoms of Female and Male OSA Patients}

As shown in Table 3, non-specific symptom of OSA, such as insomnia [difficulty in sleep initiation (48.9\% vs. $27.4 \%$, p < 0.001 ) and sleep maintenance (56.3\% vs. $40.7 \%, \mathrm{p}<0.001)$, and early morning awakening (53.4\% vs. $44.9 \%, \mathrm{p}=0.019)$ ], loss of energy, subjective poor sleep (PSQI $8.48 \pm 3.65$ in females vs. $7.47 \pm 3.37$ in males, $\mathrm{p}<0.001$ ), and depressive mood (BDI-II $15.58 \pm 9.38$ in females vs. $12.17 \pm 7.68$ in males, $\mathrm{p}<0.001$ ) were reported more frequently in females than in males. Whereas males complained about OSA classic symptoms, such as snoring, witnessed apnea, and excessive daytime sleepiness, more frequently than females did, irritability did not show a meaningful statistical difference between the two groups.

\section{Sleep Parameters of Female and Male OSA Patients}

TST, WASO (\%), and SE of polysomnography were similar in female and male OSA patients. Sleep onset latency was longer in females than in males $(15.45 \pm 22.14$ min vs. $10.11 \pm 15.14$ min, $\mathrm{p}<0.001)$. The total AHI was lower in females than in males $(26.25 \pm 18.04 / \mathrm{h}$ vs. $33.36 \pm 21.01 / \mathrm{h}, \mathrm{p}<0.001)$, but AHI was similar between the two groups of those older than 70 years (Fig. 1B, Table 2). The proportion of OSA severity was different between the two groups. Nearly $80 \%$ of males were categorized as having moderate or severe OSA, whereas mild, moderate, and severe OSA were evenly distributed among females. The arousal index and respiratory events $(\mathrm{p}<0.001)$ were lower in females than in males, but spontaneous arousal index $(\mathrm{p}<0.001)$ was higher in females (Table 4).

\section{DISCUSSION}

This study described the demographics, clinical manifestations, and polysomnographic characteristics in a group of females and males with OSA. Age had a significant effect on the distinction of OSA prevalence and severity between gender groups. Female OSA patients were older in general and showed less severe OSA manifestations than males did until they reached 70 in age, as was in concordance with other articles [8,18-21]. The ratio of OSA prevalence between males and females showed a gradual decrease as the age went up. Specifically, the ratio was about 10:1 in the 30s, 6:1 in the 40s, 3-4:1 in the 50s, $2: 1$ in the 60s, and almost 1:1 in the 70s. Moreover, after the age of 70 years, OSA severity was similar between the two groups. In other words, moderate to severe OSA is more prevalent in elderly women. Sex hormones, such as estrogen and progesterone, are thought to protect against OSA in females, which can be accounted for by increasing genioglossus muscle tone and preventing collapse of the upper airway in the premenopausal period [7,22-24]. Row-

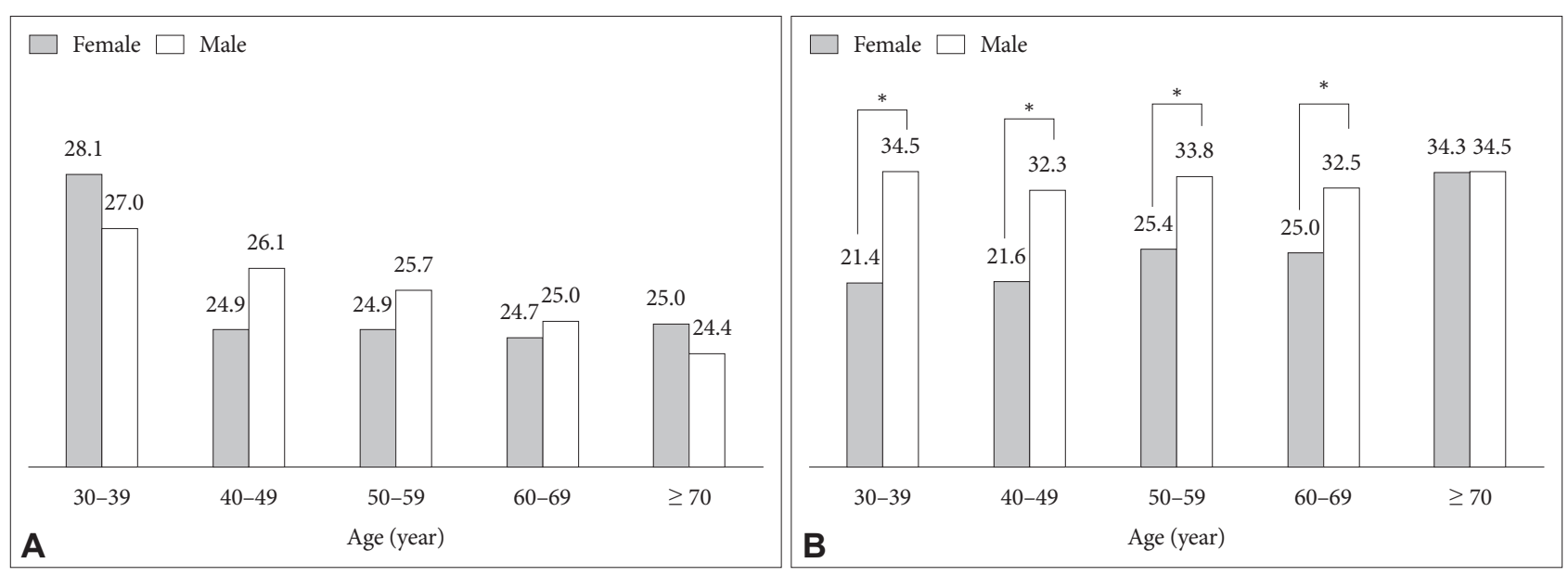

Fig. 1. The distribution of $\mathrm{BMI}$ and $\mathrm{AHI}$ according to age group. A: The distribution of BMl according to age group was not significantly different between the two groups (all $p>0.05$ ). B: AHI was lower in females under the age of 70 years. However, in group of age older than 70 years, $\mathrm{AHI}$ was similar between the two groups. ${ }^{*} \mathrm{p}<0.05$. BMI: body mass index, AHI: apnea-hypopnea index. 
Table 1. Demographics of female and male obstructive sleep apnea patients

\begin{tabular}{|c|c|c|c|c|}
\hline Variables & Total $(\mathrm{n}=1224)$ & Female $(n=277)$ & Male $(\mathrm{n}=947)$ & $\mathrm{p}$-value \\
\hline Age, years & $54.30 \pm 11.75$ & $59.62 \pm 11.16$ & $52.75 \pm 11.46$ & $<0.001$ \\
\hline $30-39$ years & $167(13.6)$ & $15(5.4)$ & $152(16.0)$ & \\
\hline $40-49$ years & $242(19.8)$ & $34(12.3)$ & $208(22.0)$ & \\
\hline $50-59$ years & $403(32.9)$ & $84(30.3)$ & $319(33.7)$ & \\
\hline $60-69$ years & $294(24.0)$ & $92(33.2)$ & $202(21.3)$ & \\
\hline$\geq 70$ years & $118(9.6)$ & $52(18.8)$ & $66(7.0)$ & \\
\hline \multicolumn{5}{|l|}{ Education } \\
\hline$\leq 12$ years & $416(34.0)$ & $167(60.3)$ & $249(26.3)$ & $<0.001$ \\
\hline$\geq 13$ years & $808(66.0)$ & $110(39.7)$ & $698(73.7)$ & \\
\hline Body mass index, $\mathrm{kg} / \mathrm{m}^{2}$ & $25.60 \pm 3.45$ & $25.01 \pm 3.96$ & $25.77 \pm 3.27$ & 0.004 \\
\hline$\geq 30 \mathrm{~kg} / \mathrm{m}^{2}$ & $118(9.6)$ & $35(12.6)$ & $83(8.8)$ & 0.055 \\
\hline Neck circumference-standing, $\mathrm{cm}^{*}$ & $38.41 \pm 3.77$ & $34.52 \pm 2.77$ & $39.46 \pm 3.29$ & $<0.001$ \\
\hline Neck circumference-lying, cm* & $39.03 \pm 3.52$ & $35.02 \pm 2.78$ & $40.12 \pm 2.86$ & $<0.001$ \\
\hline Waist circumference, $\mathrm{cm}^{*}$ & $91.82 \pm 9.29$ & $85.95 \pm 9.80$ & $93.41 \pm 8.48$ & $<0.001$ \\
\hline Hip circumference, $\mathrm{cm}^{*}$ & $96.45 \pm 6.66$ & $94.31 \pm 6.68$ & $97.02 \pm 6.54$ & $<0.001$ \\
\hline Waist-hip ratio* & $0.95 \pm 0.09$ & $0.91 \pm 0.07$ & $0.96 \pm 0.10$ & $<0.001$ \\
\hline Alcohol & $732(61.2)$ & $61(23.2)$ & $671(71.8)$ & $<0.001$ \\
\hline \multicolumn{5}{|l|}{ Smoking } \\
\hline Ex-smoker & $325(26.7)$ & $4(1.5)$ & $321(34.0)$ & $<0.001$ \\
\hline None & $687(56.5)$ & $262(96.0)$ & $425(45.1)$ & \\
\hline Current & $204(16.8)$ & $7(2.6)$ & $197(20.9)$ & \\
\hline \multicolumn{5}{|l|}{ Comorbidities } \\
\hline Hypertension & $356(29.1)$ & $86(31.0)$ & $270(28.5)$ & 0.414 \\
\hline Diabetes mellitus & $146(11.9)$ & $30(10.8)$ & $116(12.2)$ & 0.522 \\
\hline Cardiovascular disease & $130(10.6)$ & $28(10.1)$ & $102(10.9)$ & 0.753 \\
\hline Cerebrovascular disease $^{\dagger}$ & $8(0.7)$ & $1(0.4)$ & $7(0.6)$ & 0.691 \\
\hline Hyperlipidemia & $49(4.0)$ & $15(5.4)$ & $34(3.6)$ & 0.173 \\
\hline Respiratory disease & $40(3.3)$ & $8(2.9)$ & $32(3.4)$ & 0.686 \\
\hline Otolaryngologic history ${ }^{\dagger}$ & $17(1.4)$ & $5(1.8)$ & $12(1.3)$ & 0.558 \\
\hline Use of hypnotics and sedatives & $140(12.1)$ & $56(22.3)$ & $84(9.3)$ & $<0.001$ \\
\hline
\end{tabular}

Data are presented as $\mathrm{n}(\%)$ or mean \pm standard deviation.

*Total numbers are 478 (female: male $=102: 376$ ). ${ }^{\dagger}$ Analyzed by Fisher's exact test.

Table 2. BMI and AHI distribution according to age group

\begin{tabular}{|c|c|c|c|c|c|c|}
\hline \multirow{2}{*}{ Age } & \multicolumn{3}{|c|}{ BMI } & \multicolumn{3}{|c|}{$\mathrm{AHI}$} \\
\hline & Female & Male & p-value & Female & Male & p-value \\
\hline 30-39 years* & $28.10 \pm 4.44$ & $26.97 \pm 4.49$ & $0.131^{*}$ & $21.44 \pm 20.49$ & $34.47 \pm 25.78$ & $0.017^{*}$ \\
\hline $40-49$ years & $24.87 \pm 4.06$ & $26.14 \pm 3.27$ & 0.089 & $21.58 \pm 16.61$ & $32.29 \pm 21.10$ & 0.002 \\
\hline $50-59$ years & $24.88 \pm 4.00$ & $25.70 \pm 2.77$ & 0.077 & $25.39 \pm 18.12$ & $33.83 \pm 19.08$ & $<0.001$ \\
\hline $60-69$ years & $24.70 \pm 3.69$ & $25.05 \pm 2.68$ & 0.421 & $25.00 \pm 15.83$ & $32.51 \pm 19.09$ & 0.001 \\
\hline$\geq 70$ years & $24.99 \pm 3.91$ & $24.37 \pm 2.69$ & 0.310 & $34.32 \pm 19.84$ & $34.51 \pm 23.24$ & 0.962 \\
\hline
\end{tabular}

Data are presented as mean \pm standard deviation.

*Analyzed by Mann-Whitney test.

BMI: body mass index, AHI: apnea-hypopnea index. 
ley et al. [25] supported that estrogen/progesterone decrease the apneic threshold to $\mathrm{CO}_{2}$ and the sensitivity to hypercapnia or hypoxia, leading to a decrease in breathing instability, hence explaining the fact that sleep apnea is less prevalent in menstruating females. The average age of Korean females with menopause is $48^{-49}$ years [26], and the mean age of all females, in-

Table 3. Presenting sleep related symptoms and sleep questionnaires of female and male obstructive sleep apnea patients

\begin{tabular}{lccrrr}
\hline \multicolumn{1}{c}{ Variables } & Total $(\mathrm{n}=1224)$ & Female $(\mathrm{n}=277)$ & Male $(\mathrm{n}=947)$ & $\mathrm{p}$-value \\
\hline Snoring & $1117(91.8)$ & $239(87.2)$ & $878(93.1)$ & 0.002 \\
Witnessed sleep apnea & $825(67.8)$ & $128(46.7)$ & $697(73.9)$ & $<0.001$ \\
Sleep initiation problem & $388(32.2)$ & $132(48.9)$ & $256(27.4)$ & $<0.001$ \\
Sleep maintenance problem & $533(44.2)$ & $152(56.3)$ & $381(40.7)$ & $<0.001$ \\
Early awakening problem & $547(46.8)$ & $135(53.4)$ & $412(44.9)$ & 0.019 \\
Loss of energy & $349(28.7)$ & $109(39.8)$ & $240(25.4)$ & $<0.001$ \\
Irritability & $145(11.9)$ & $32(11.7)$ & $113(12.0)$ & 0.896 \\
Insomnia severity index & $11.39 \pm 6.51$ & $12.94 \pm 7.16$ & $10.95 \pm 6.24$ & $<.47 \pm 3.37$ & $<0.001$ \\
Pittsburgh sleep quality index & $7.69 \pm 3.45$ & $8.48 \pm 3.65$ & $8.36 \pm 5.62$ & $10.55 \pm 4.90$ & $<0.001$ \\
ESS & $10.06 \pm 5.15$ & $87(31.5)$ & $467(49.5)$ & $<0.001$ \\
$\quad$ Excessive daytime sleepiness (ESS $>10)$ & $554(45.4)$ & $15.58 \pm 9.38$ & $12.17 \pm 7.68$ & $<0.001$ \\
Beck Depression Inventory-II & $12.93 \pm 8.21$ & &
\end{tabular}

Data are presented as $\mathrm{n}(\%)$ or mean \pm standard deviation.

ESS: Epworth Sleepiness Scale.

Table 4. Polysomnography parameters of female and male OSA patients

\begin{tabular}{|c|c|c|c|c|}
\hline Variables & Total $(\mathrm{n}=1224)$ & Female $(\mathrm{n}=277)$ & Male $(\mathrm{n}=947)$ & p-value \\
\hline Total sleep time, min & $360.65 \pm 63.22$ & $364.61 \pm 67.58$ & $359.49 \pm 61.88$ & 0.236 \\
\hline Sleep latency, min & $11.31 \pm 17.11$ & $15.45 \pm 22.14$ & $10.11 \pm 15.14$ & $<0.001$ \\
\hline Wakefulness sleep onset, \% & $16.20 \pm 11.23$ & $16.24 \pm 11.71$ & $16.18 \pm 11.10$ & 0.938 \\
\hline Sleep efficiency, \% & $81.84 \pm 11.93$ & $81.11 \pm 12.93$ & $82.06 \pm 11.61$ & 0.243 \\
\hline \multicolumn{5}{|l|}{ Sleep stages } \\
\hline N1 sleep, $\%$ & $24.67 \pm 14.18$ & $19.36 \pm 12.02$ & $26.23 \pm 14.40$ & $<0.001$ \\
\hline N2 sleep, $\%$ & $52.88 \pm 12.24$ & $56.15 \pm 11.49$ & $51.93 \pm 12.30$ & $<0.001$ \\
\hline N3 sleep, $\%$ & $2.60 \pm 4.68$ & $4.38 \pm 6.10$ & $2.08 \pm 4.04$ & $<0.001$ \\
\hline REM sleep, \% & $19.87 \pm 6.81$ & $20.10 \pm 7.11$ & $19.81 \pm 6.72$ & 0.528 \\
\hline AHI, /h & $31.75 \pm 20.58$ & $26.25 \pm 18.04$ & $33.36 \pm 21.01$ & $<0.001$ \\
\hline $\mathrm{AHI}_{\mathrm{REM}}, / \mathrm{h}$ & $32.94 \pm 20.87$ & $33.55 \pm 21.20$ & $32.76 \pm 20.78$ & 0.581 \\
\hline $\mathrm{AHI}_{\mathrm{NREM}}, / \mathrm{h}$ & $29.99 \pm 21.27$ & $23.25 \pm 18.51$ & $31.96 \pm 21.62$ & $<0.001$ \\
\hline $\mathrm{AHI}_{\text {supine }}, / \mathrm{h}$ & $46.14 \pm 26.21$ & $35.53 \pm 22.74$ & $49.24 \pm 26.36$ & $<0.001$ \\
\hline $\mathrm{AHI}_{\text {lateral }}, / \mathrm{h}$ & $15.30 \pm 19.64$ & $12.27 \pm 16.62$ & $16.19 \pm 20.36$ & $<0.001$ \\
\hline \multicolumn{5}{|l|}{ OSA severity } \\
\hline Mild $(5 \leq$ AHI < $15 / h)$ & $271(22.1)$ & $89(32.1)$ & $182(19.2)$ & $<0.001$ \\
\hline Moderate $(15 \leq \mathrm{AHI}<30 / \mathrm{h})$ & $423(34.6)$ & $106(38.3)$ & $317(33.5)$ & \\
\hline Severe $(\mathrm{AHI} \geq 30 / \mathrm{h})$ & $530(43.3)$ & $82(29.6)$ & $448(47.4)$ & \\
\hline Oxygen desaturation index & $26.19 \pm 19.88$ & $21.40 \pm 17.50$ & $27.60 \pm 20.32$ & $<0.001$ \\
\hline $\mathrm{AI}, / \mathrm{h}$ & $29.86 \pm 17.57$ & $23.69 \pm 13.37$ & $27.60 \pm 20.32$ & $<0.001$ \\
\hline Respiratory AI, /h & $21.80 \pm 17.33$ & $15.8 \pm 12.97$ & $23.74 \pm 17.96$ & $<0.001$ \\
\hline Spontaneous AI, /h & $3.71 \pm 3.74$ & $4.48 \pm 4.24$ & $3.48 \pm 3.55$ & $<0.001$ \\
\hline
\end{tabular}

Data are presented as $\mathrm{n}(\%)$ or mean \pm standard deviation.

REM: rapid eye movement, $\mathrm{AHI}$ : apnea-hypopnea index, $\mathrm{AHI}_{\mathrm{REM}}$ : $\mathrm{AHI}$ during REM sleep, $\mathrm{AHI}_{\mathrm{NREM}}$ : $\mathrm{AHI}$ during non-REM sleep, $\mathrm{AHI}_{\text {suprine: }}$ AHI during supine position, $\mathrm{AHI}_{\text {lateral: }} \mathrm{AHI}$ during lateral position, $\mathrm{AI}$ : arousal index, OSA: obstructive sleep apnea. 
cluding those in this study, was 59.3 years. Although individual information on the menopausal status of females could not be investigated, we infer that a large portion of the females in our study might have been postmenopausal and that the incidence of OSA in females might increase after menopause.

Obesity is an important risk factor for the development of OSA and is related to the severity of OSA, because it may reduce upper airway diameter and increase its collapsibility $[10,27,28]$. The mean BMI, which is a measurement of obesity, was $25.01 \pm 3.96$ in females and $25.77 \pm 3.28 \mathrm{~kg} / \mathrm{m}^{2}$ in males. The incidence of obesity, defined by western cutoff (BMI $\left.\geq 30 \mathrm{~kg} / \mathrm{m}^{2}\right)$, is relevant in $12.6 \%$ of females and $8.8 \%$ of males. These rates are lower than those in previous studies of western countries [29]. Our subjects still had OSA, despite having a smaller percentage of obesity defined by western criteria in our groups. These findings imply that other risk factors, namely, content of body fat and anatomical structure of the upper airway, may be responsible for development of OSA. Li et al. [30] found Far East Asian males (72\% Chinese, 20\% Japanese, and 8\% Korean subjects) were less obese in spite of a greater severity of OSA, and attributed these differences to differences in craniofacial anatomy, such as more protruded maxilla and mandibles, and less inferiorly displaced hyoid and decreased cranial base dimension. Also, under the age of 70 , females had a lower AHI than did males, even though their BMI's were not different in this study. It is well recognized that males tend to gain weight more centrally than do females, and this pattern probably results in males having more fat stored in upper-airway structures and in the abdomen than do females, whereas females have lower body fat distribution [10]. In many other studies [31], BMI and AHI were known to be relevant because of pharyngeal fat-tissue deposition.

The clinical under-diagnosis of OSA in females may result from gender differences in presenting symptom profiles to primary physicians. Females were presenting distinct symptoms with less complaining of OSA classical phenotypes, namely, snoring, witnessed apneas, and excessive daytime sleepiness. Females may consider these typical symptoms to be less socially acceptable, and as a result, they are underreported. Redline et al. [32] evaluated classic symptoms of OSA, namely, daytime somnolence, snoring, and witnessed apnea, as compared between genders. Females consistently under-recognized symptoms of snoring, gasping, and apnea. Males were 2-3 times more likely than female were to report these symptoms [32]. Quintana-Gallego et al. [8] also assessed 1166 patients (male: female ratio $=4.9: 1$ ) who fulfilled the criteria of OSA (AHI $\geq 10$ ). The frequency of snoring and daytime somnolence was similar in both genders, although witnessed apneas were more frequent in males. Fatigue, morning headaches, insomnia, and depressive mood were more common in females than in males $[8,18,32]$. Difficulty of sleep initiation and maintenance and early morning awakening were more frequently reported as a presenting complaint by females than by male. In a study of 2052 Turkish male and 775 female
OSA patients, the females reported a higher percentage of insomnia symptoms, including difficulty of initiating sleep, maintaining sleep, and early morning awakenings, than males did (34\% vs. $23 \%, \mathrm{p}<0.001$ ) [21]. These findings indicate the importance of assessing females who present with insomnia for the possibility of OSA. Many other studies have suggested that under-reporting of OSA classic symptoms, such as cessation of breathing during sleep and more non-specific symptoms, may explain the clinical under-diagnoses of OSA in females [32,33]. Previous studies reported that insomnia was accompanied in $22 \%$ to $67 \%$ of OSA patients [34-37]. The more-frequent insomnia complaints in females were consistent with higher frequencies of hypnotic agents than for males. Males complained more about witnessed apnea and daytime sleepiness. Although the pathogenesis underlying sleepiness remains to be elucidated, the presence of excessive daytime sleepiness in OSA patients has been attributed to nocturnal hypoxemia, sleep fragmentation, or both [38]. This result was supported by findings that OSA severity was significantly correlated with ESS score only in male patients and that male OSA patients had a higher oxygen desaturation index, a higher total respiratory arousal index, and a more increased N1 sleep but decreased slow-wave sleep [23] than did females. Female OSA patients might report their sleepiness more differently and emphasize fatigue, tiredness, and loss of energy more than sleepiness, whereas males complained of sleepiness [39].

The male: female ratio for OSA prevalence increased from 2.0:1 for mild OSA to 5.5:1 for severe OSA in our study. that is, females had a higher proportion of mild apnea during TST. Our findings of milder OSA in females than in males are consistent with previous studies [20]. In females, AHI during TST and nonREM (NREM) sleep were less severe than in males, but during REM stage, it was similar in both genders. O'Connor et al. [20] compared females and males for the difference between the $\mathrm{AHI}_{\mathrm{REM}}$ and $\mathrm{AHI}_{\mathrm{NREM}}$ (REM difference) and found that the difference was almost threefold greater in females than in males. Females also had a greater proportion of respiratory events during REM stage than males did. A possible explanation is gonadal hormones, such as progesterone, may play an important role in increasing upper-airway muscle tone during wakefulness and NREM sleep, but this protective effect disappears during REM sleep [11]. In the community based "Sleep and Health in Women" cohort study, repeated obstructive apnea during REM sleep was considered to be a cerebrovascular risk, defined as increased carotid artery intima thickness, at 10-year follow-up [40]. This implies that the presence of frequent obstructive apnea during REM sleep might be considered in early diagnosis and continuous positive airway pressure treatment of OSA. In our female OSA patients, greater reported difficulty in sleep initiation and maintenance might have been more associated with longer sleep onset latency and spontaneous arousal index, respectively, than in males.

Some limitations should be noted. The individual information 
on menopausal status was not collected. Second, our study sample was evaluated retrospectively. Third, the study at a tertiary care center was associated with referral bias and limited generalizability. Nevertheless, this study is relevant in presenting the gender-different distribution of OSA prevalence according to ages in a relatively large number of Korean patients.

In conclusion, our findings suggest that females have a different OSA phenotype. First, they are symptomatic at relatively low $\mathrm{AHI}$ and have a distinct symptom profile. Factors ascribed to gender differences in the recognition of OSA include differences in how females present symptoms as compared to males. Female OSA patients are more likely complain of non-specific symptoms of OSA, including insomnia, subjective poor sleep, and depressive mood. The result of a longer sleep-onset latency and a higher spontaneous arousal index in females than in males may suggest that female OSA patients may have an insomnia component. Second, female OSA patients are older, and moderate to severe OSA is more prevalent in elderly females. Third, females OSA patients have frequent obstructive apnea during REM, which is considered to be a vascular risk factor. These findings imply that female OSA might be misdiagnosed as insomnia, depression, or chronic fatigue syndrome, and elderly female patients complaining of sleep disorders might have OSA prevalence. Therefore, primary physicians should be informed about the different clinical phenotype of female OSA patients and thus make early diagnoses and appropriate interventions in females with undiagnosed OSA in order to lessen the adverse health outcomes.

\section{Acknowledgments}

This research is supported by Samsung Biomedical Research Institute grant (OTC1190671).

\section{Conflicts of Interest}

The authors have no financial conflicts of interest.

\section{Authors' Contribution}

Conceptulization: Pyun SY. Supervision: Joo EY. Writing_original draft: Pyun SY, Choi SJ. Writing-review \& editing: Pyun SY, Choi SJ, Jo H, Hwang Y, Cho JW.

\section{REFERENCES}

1. Young T, Palta M, Dempsey J, Skatrud J, Weber S, Badr S. The occurrence of sleep-disordered breathing among middle-aged adults. N Engl J Med 1993;328:1230-5.

2. Durán J, Esnaola S, Rubio R, Iztueta A. Obstructive sleep apnea-hypopnea and related clinical features in a population-based sample of subjects aged 30 to 70 yr. Am J Respir Crit Care Med 2001;163:685-9.

3. Ip MS, Lam B, Tang LC, Lauder IJ, Ip TY, Lam WK. A community study of sleep-disordered breathing in middle-aged Chinese women in Hong Kong: prevalence and gender differences. Chest 2004;125:127-34.

4. Sharma SK, Kumpawat S, Banga A, Goel A. Prevalence and risk factors of obstructive sleep apnea syndrome in a population of Delhi, India. Chest 2006;130:149-56.

5. Nakayama-Ashida Y, Takegami M, Chin K, Sumi K, Nakamura T, Takahashi K, et al. Sleep-disordered breathing in the usual lifestyle setting as detected with home monitoring in a population of working men in Japan. Sleep 2008;31:419-25.
6. Kim J, In K, Kim J, You S, Kang K, Shim J, et al. Prevalence of sleepdisordered breathing in middle-aged Korean men and women. Am J Respir Crit Care Med 2004;170:1108-13.

7. Young T, Finn L, Austin D, Peterson A. Menopausal status and sleepdisordered breathing in the Wisconsin Sleep Cohort Study. Am J Respir Crit Care Med 2003;167:1181-5.

8. Quintana-Gallego E, Carmona-Bernal C, Capote F, Sánchez-Armengol A, Botebol-Benhamou G, Polo-Padillo J, et al. Gender differences in obstructive sleep apnea syndrome: a clinical study of 1166 patients. Respir Med 2004;98:984-9.

9. Mohsenin V. Effects of gender on upper airway collapsibility and severity of obstructive sleep apnea. Sleep Med 2003;4:523-9.

10. Millman RP, Carlisle CC, McGarvey ST, Eveloff SE, Levinson PD. Body fat distribution and sleep apnea severity in women. Chest 1995;107: 362-6.

11. Popovic RM, White DP. Upper airway muscle activity in normal women: influence of hormonal status. J Appl Physiol (1985) 1998;84:1055-62.

12. Lévy P, Kohler M, McNicholas WT, Barbé F, McEvoy RD, Somers VK, et al. Obstructive sleep apnoea syndrome. Nat Rev Dis Primers 2015;1: 15015.

13. Iber C, Ancoli-Israel S, Chesson AL, Quan SF. The AASM manual for the scoring of sleep and associated events: rules, terminology, and technical specification. 1th ed. Westchester, IL: American Academy of Sleep Medicine 2007.

14. Johns MW. A new method for measuring daytime sleepiness: the Epworth Sleepiness Scale. Sleep 1991;14:540-5.

15. Bastien $\mathrm{CH}$, Vallières A, Morin CM. Validation of the insomnia severity index as an outcome measure for insomnia research. Sleep Med 2001;2:297-307.

16. Buysse DJ, Reynolds CF III, Monk TH, Berman SR, Kupfer DJ. The Pittsburgh sleep quality index: a new instrument for psychiatric practice and research. Psychiatry Res 1989;28:193-213.

17. Beck AT, Steer RA, Brown GK. Manual for the Beck Depression Inventory-II. San Antonio, TX: Psychological Corporation 1996.

18. Larsson LG, Lindberg A, Franklin KA, Lundbäck B. Gender differences in symptoms related to sleep apnea in a general population and in relation to referral to sleep clinic. Chest 2003;124:204-11.

19. Vagiakis E, Kapsimalis F, Lagogianni I, Perraki H, Minaritzoglou A, Alexandropoulou K, et al. Gender differences on polysomnographic findings in Greek subjects with obstructive sleep apnea syndrome. Sleep Med 2006;7:424-30.

20. O'Connor C, Thornley KS, Hanly PJ. Gender differences in the polysomnographic features of obstructive sleep apnea. Am J Respir Crit Care Med 2000;161:1465-72.

21. Basoglu OK, Tasbakan MS. Gender differences in clinical and polysomnographic features of obstructive sleep apnea: a clinical study of 2827 patients. Sleep Breath 2018;22:241-9.

22. Gabbay IE, Lavie P. Age- and gender-related characteristics of obstructive sleep apnea. Sleep Breath 2012;16:453-60.

23. Stanescu D, Veriter C. A model of obstructive sleep apnea in normal humans. Am J Respir Crit Care Med 2001;163:1031.

24. Ayub S, Won CHJ. Obstructive sleep apnea in women. J Sleep Med 2019;16:75-80.

25. Rowley JA, Zhou XS, Diamond MP, Badr MS. The determinants of the apnea threshold during NREM sleep in normal subjects. Sleep 2006;29: 95-103.

26. Ku SY, Kang JW, Kim H, Ku PS, Lee SH, Suh CS, et al. Regional differences in age at menopause between Korean-Korean and Korean-Chinese. Menopause 2004;11:569-74.

27. Lim YH, Choi J, Kim KR, Shin J, Hwang KG, Ryu S, et al. Sex-specific characteristics of anthropometry in patients with obstructive sleep apnea: neck circumference and waist-hip ratio. Ann Otol Rhinol Laryngol 2014;123:517-23.

28. Cho JH, Choi JH, Suh JD, Ryu S, Cho SH. Comparison of anthropometric data between Asian and Caucasian patients with obstructive 
sleep apnea: a meta-analysis. Clin Exp Otorhinolaryngol 2016;9:1-7.

29. Young T, Shahar E, Nieto FJ, Redline S, Newman AB, Gottlieb DJ, et al. Predictors of sleep-disordered breathing in community-dwelling adults: the Sleep Heart Health Study. Arch Intern Med 2002;162:893-900.

30. Li KK, Kushida C, Powell NB, Riley RW, Guilleminault C. Obstructive sleep apnea syndrome: a comparison between Far-East Asian and white men. Laryngoscope 2000;110:1689-93.

31. Shelton KE, Woodson H, Gay S, Suratt PM. Pharyngeal fat in obstructive sleep apnea. Am Rev Respir Dis 1993;148:462-6.

32. Redline S, Kump K, Tishler PV, Browner I, Ferrette V. Gender differences in sleep disordered breathing in a community-based sample. Am J Respir Crit Care Med 1994;149:722-6.

33. Kapsimalis F, Kryger MH. Gender and obstructive sleep apnea syndrome, part 1: clinical features. Sleep 2002;25:412-9.

34. Chung KF. Insomnia subtypes and their relationships to daytime sleepiness in patients with obstructive sleep apnea. Respiration 2005;72:460-5.

35. Al-Jawder SE, Bahammam AS. Comorbid insomnia in sleep-related breathing disorders: an under-recognized association. Sleep Breath 2012; 16:295-304.

36. Krakow B, Melendrez D, Ferreira E, Clark J, Warner TD, Sisley B, et al. Prevalence of insomnia symptoms in patients with sleep-disordered breathing. Chest 2001;120:1923-9.

37. Lichstein KL, Justin Thomas S, Woosley JA, Geyer JD. Co-occurring insomnia and obstructive sleep apnea. Sleep Med 2013;14:824-9.

38. Colt HG, Haas H, Rich GB. Hypoxemia vs sleep fragmentation as cause of excessive daytime sleepiness in obstructive sleep apnea. Chest 1991; 100:1542-8.

39. Chervin RD. Sleepiness, fatigue, tiredness, and lack of energy in obstructive sleep apnea. Chest 2000;118:372-9.

40. Ljunggren M, Lindberg E, Franklin KA, Öhagen P, Larsson M, Theorell-Haglöw J, et al. Obstructive sleep apnea during rapid eye movement sleep is associated with early signs of atherosclerosis in women. Sleep 2018;41. 